\title{
Laterally coupled nanowire lasers:
}

\section{Bifurcations, dynamics and high-speed potential}

\author{
Antonio Hurtado, Dimitars Jevtics \\ Institute of Photonics \\ University of Strathclyde \\ Glasgow, UK \\ antonio.hurtado@strath.ac.uk
}

\author{
Mike Adams, Ian Henning \\ School of Computer Science and Electronic \\ Engineering \\ University of Essex \\ Colchester, UK
}

\begin{abstract}
Regions of stability in two laterally-coupled InP nanowire lasers are analysed in terms of their separation, difference in resonant frequencies and pumping rate. The frequency of periodic oscillations for realistic laser separations and pumping is estimated to be of order 100- $1000 \mathrm{GHz}$
\end{abstract}

\section{INTRODUCTION}

The study of arrays of laterally coupled semiconductor lasers that was focussed initially on production of high-power sources has turned more recently to aspects of nonlinear dynamics that are of fundamental importance for developing new applications (see [1] and references therein). In the case of nanolasers, two-element arrays of photonic crystal vertical cavity surface-emitting lasers (PC-VCSELs) have exhibited bistability, excitability, and selfpulsations [2], spontaneous mirror-symmetry breaking through a pitchfork bifurcation [3], and modal switching between in-phase and out-of-phase modes [4], An advantage of PC-VCSELs is that precise control of the coupling between adjacent lasers can be achieved by changing the diameter of the hole in the 'photonic barrier' between emitters [5]. In the case of nanowire (NW) lasers, coupling between two elements must be performed by nanoprobe manipulation [6] which does not allow very accurate control. However, practical routes to improving NW laser positioning accuracy are emerging, for example novel transfer printing techniques [7], and thus it is timely to begin theoretical exploration of the dynamics of evanescently-coupled NW lasers.

\section{THEORY}

Coupling strength in laser arrays is measured by the product of coupling rate $\kappa$ and photon lifetime $\tau_{p}$. Strong coupling is typically characterised by $\kappa \tau_{p}>1$ (as in, e.g., [4] with values of 8 and 12). Here we consider the case of weak coupling in evanescentlycoupled pairs of indium phosphide (InP) NW lasers. Simple but accurate approximations for the bifurcations separating regions of stable and unstable dynamics derived in [8] will be used to show that weakly-coupled NW laser arrays can be expected to exhibit a wide range of behaviour including periodicity with high frequencies, complex dynamics and chaos.
We have calculated $\kappa$ for InP NW lasers placed on a silica substrate (see insets in Fig. 1) for a range of edge-to-edge separations from $100 \mathrm{~nm}$ to $1000 \mathrm{~nm}$ using finite difference eigenmode (FDE) software. For NW hexagonal core diameter of $300 \mathrm{~nm}$ and wavelength $880 \mathrm{~nm}$ [9] the only guided modes in each individual $\mathrm{NW}$ are $\mathrm{HE}_{11 \mathrm{a}}, \mathrm{HE}_{11 \mathrm{~b}}$ and $\mathrm{TE}_{01}$, following the naming notation used for circular waveguides; the subscripts ' $a$ ', 'b' on the hybrid modes distinguish the polarisations. Values of the photon lifetime are found for each mode from $\tau_{p}=\left(v_{g} \Gamma g_{t h}\right)^{-1 / 2}$, where $v_{g}$ is the group velocity, $\Gamma$ is the mode confinement factor, and $g_{t h}$ is the gain per unit length at threshold. Values of the latter two quantities are found from simulations in [9] for $5 \mu \mathrm{m}$-long InP NW lasers.

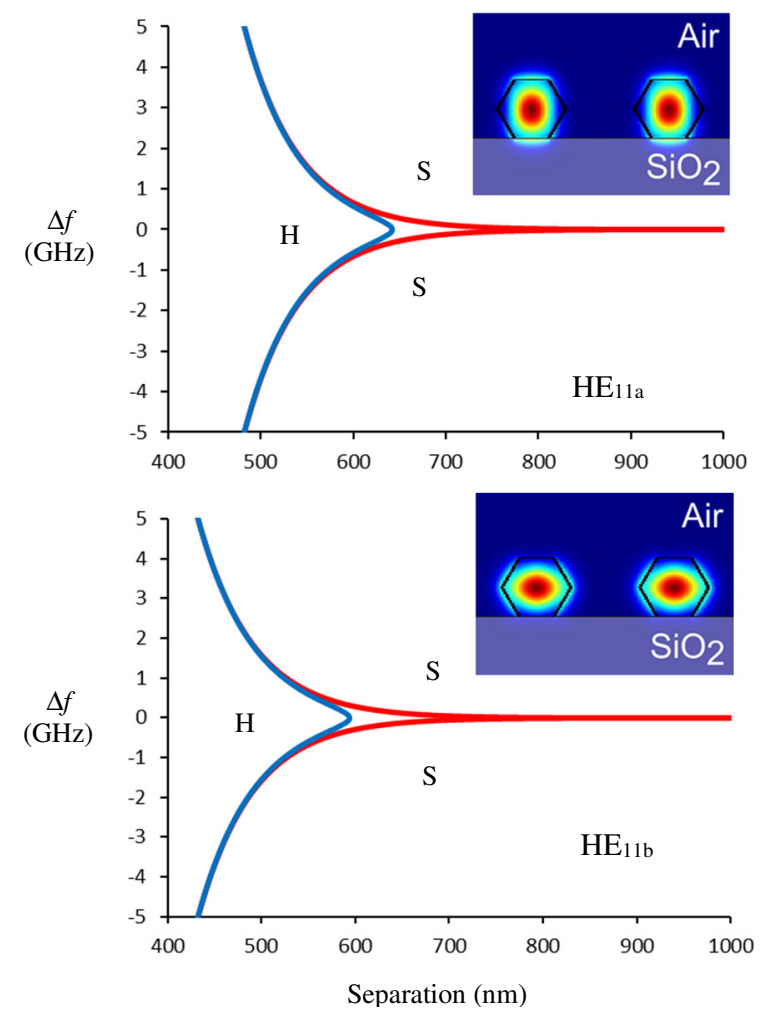

Fig. 1. Saddle-node (S) and Hopf $(\mathrm{H})$ bifurcations for the $\mathrm{HE}_{11 \mathrm{a}}$ and $\mathrm{HE}_{11 \mathrm{~b}}$ modes for $\alpha_{H}=3, \tau_{n}=1 \mathrm{~ns}$ and $Q=2$. The insets show the coupled NW structure and mode intensity profiles in each case. 


\section{RESULTS}

Fig.1 shows the calculated Hopf and saddle-node bifurcations using the approximations from [8] as plots of the difference in resonant frequencies of the two lasers versus the edge-to-edge separation. For this calculation the linewidth enhancement factor $\alpha_{H}=3$, recombination lifetime $\tau_{N}=1 \mathrm{~ns}$, and the normalised pumping rate $Q=2$ [8]. The regions of stability where the two lasers are phase-locked in the antiphase normal mode lie between these bifurcations. Outside these regions various forms of instability including periodic oscillation, chaos and complex dynamics are found [10]. The regions of stable locked behaviour are very small, mainly close to zero frequency offset between the lasers. Since the fabrication process implies an unavoidable variation in NW laser cavity length and hence resonant frequency, this means that they are unlikely to ever show stable locking; under almost all operating conditions they will exhibit various forms of unstable dynamics under conditions of weak coupling.

In the regions of instability for zero frequency offset, a first-order approximation for the frequency $f$ of periodic oscillations is given by [11]

$$
f=\frac{1}{2 \pi} \sqrt{4 \kappa^{2}+\frac{Q-1}{\tau_{N} \tau_{p}}}
$$

The first term in the square root refers to the frequency of energy exchange between the NW lasers, whilst the second term corresponds to the relaxation oscillation frequency (ROF). Plots of the frequency $f$ versus $2 d$ calculated from equation (1) are shown in Fig. 2 for the $\mathrm{HE}_{11 \mathrm{a}}, \mathrm{HE}_{1 \mathrm{lb}}$ and $\mathrm{TE}_{01}$ modes using the same parameters as for Fig. 1.

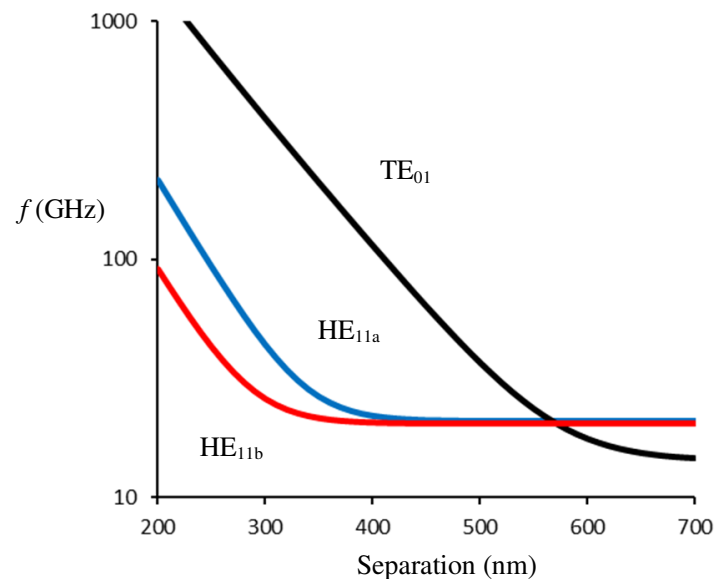

Fig. 2. Frequency of periodic oscillations at zero frequency offset for the $\mathrm{HE}_{11 \mathrm{a}}, \mathrm{HE}_{11 \mathrm{~b}}$ and $\mathrm{TE}_{01}$ modes.

The very high frequencies of order $100-1000 \mathrm{GHz}$ that are predicted for lower separations in Fig. 2 might offer the potential for ultra-high-frequency modulation in these coupled NW lasers, based on a different physical mechanism to previous reports of modulation of nanolasers at frequencies above $100 \mathrm{GHz}$ [13]. If out-of-phase modulation is used, the modulation resonance frequency of laterally-coupled lasers is given by $\kappa \pi$ [12]. We recognise this as the case of equation (1) when the ROF term is neglected, so the results of Fig. 2 for lower separations are a good guide to potential modulation speeds.

\section{CONCLUSION}

Approximations for the Hopf and saddle-node bifurcations that determine nonlinear dynamics of evanescently-coupled semiconductor laser arrays have been applied to pairs of InP NW lasers positioned in lateral proximity on a silica substrate. The bifurcations for coupling between the lowest-order modes, $\mathrm{HE}_{11 \mathrm{a}}$ and $\mathrm{HE}_{11 \mathrm{~b}}$, define very small regions of stable locked operation and large regions of instability. An estimate of the frequency of periodic oscillations in this system yields high values for low separations (but still lying within the weak coupling regime). Coupled arrays of NW lasers might therefore offer the possibility of ultrahigh frequency modulation beyond the limit of the relaxation oscillation frequency. Further theoretical modelling is needed to explore the nature of the dynamics as functions of NW laser frequency offset, spatial separation and operating conditions.

\section{REFERENCES}

[1] N. Blackbeard, S. Wieczorek, H. Erzgräber, and P. S. Dutta, "From synchronization to persistent optical turbulence in laser arrays," Physica D, vol. 286-287, pp. 43-58, Oct. 2014.

[2] M. Brunstein et al, "Excitability and self-pulsing in a photonic crystal nanocavity," Phys. Rev. A, vol. 85, art. no. 031803, March 2012.

[3] P. Hamel et al, "Spontaneous mirror-symmetry breaking in coupled photonic-crystal nanolasers," Nat. Photonics, vol. 9, pp. 311-315, May 2015.

[4] M. Marconi, J. Javaloyes, F. Raineri, J. A. Levenson, and A. M. Yacomotti, "Asymmetric mode scattering in strongly coupled photonic crystal nanolasers," Opt. Lett., vol. 41, pp. 5628-5631, Dec. 2016.

[5] S. Haddadi et al, "Photonic molecules: tailoring the coupling strength and sign," Opt. Express, vol. 22, 12359-12368, May 2014

[6] H. Xu et al, "Single-mode lasing of GaN nanowire-pairs," Appl. Phys. Lett., vol. 101, art. no. 113106, Sept. 2012.

[7] A. Hurtado et al, "Transfer printing of semiconductor nanowire lasers," IET Optoelectron., vol. 12, pp. 30-35 Feb. 2018.

[8] M. J. Adams, N. Q. Li, B. R. Cemlyn, H. Susanto and I. D. Henning, "Effects of detuning, gain-guiding, and index antiguiding on the dynamics of two laterally coupled semiconductor lasers," Phys. Rev. A, vol. 95, art. no. 053869, May 2017.

[9] D. Saxena et al, "Mode profiling of semiconductor nanowire lasers," Nano Lett., vol. 15, pp. 5342-5348, August 2015.

[10] N. Q. Li, H. Susanto, B. R. Cemlyn, I. D. Henning and M. J. Adams, "Nonlinear dynamics of solitary and optically injected two-element laser arrays with four different waveguide structures: a numerical study," Opt. Express, vol. 26, pp. 47514765, Feb. 2018.

[11]H. G. Winful and S. S. Wang, "Stability of phase locking in coupled semiconductor-laser arrays," Appl. Phys. Lett., vol. 53, pp.1894-1896, Nov. 1988

[12] G.A. Wilson, R.K. DeFreez and H.G. Winful, IEEE J. Quantum Electron., "Modulation of phased-array semiconductor lasers at K-band frequencies," vol. 27, pp. 1696-1704, June 1991.

[13]H. Altug, D. Englund, and J. Vuckovic, "Ultrafast photonic crystal nanocavity laser," Nat. Phys., vol. 2, pp. 484-488, July 2006. 